\title{
Visual Servoing for Constrained Robots: A New Complete Theoretical Framework and its Experimental Validation
}

\author{
E.C Dean- Leon, L.G. Garc a- Valdovinos and \\ V. Parra-Vega* \\ Mechatronics Division \\ CINVESTAV-IPN \\ IPN 2508,Sn P. Zacatenco, Mexico, D.F.,7300. \\ edean, lgarcia, vparra@cinvestav.mx
}

\author{
A. Espinosa-Romero \\ Faculty of Mathematics \\ Universidad Autonoma de Yucatan \\ Merida, Yucatan. Mexico \\ eromero@tunku.uady.mx
}

\begin{abstract}
The theoretical framework and experimental validation of a new image-based position-force control is presented in this paper. This scheme produces simultaneous convergence of the constrained visual position and the contact force between the end-effector and the constraint surface. Camera, robot and jacobian parameters are considered uncertain. This approach is based on a new formulation of the orthogonalization principle used in force control, coined here visual orthogonalization principle. This allows, under the framework of passivity, to yield a synergetic scheme that fuses accordingly camera, encoder and force sensor signals. Furthermore, notice that visual servoing contact tasks are characterized by slow motion, and typically with velocity reversals along the constrained surface due actual technological limitations of the camera, thus, important problems of friction at the joint and contact point arise. Therefore, in this paper, compensation of dynamic joint friction and viscous contact friction are also studied. In order to prove the effectiveness of the theoretical scheme, a Linux-RTAI real-time OS experimental system is used to obtain a direct-drive robot manipulator equipped with six axis JR3 force sensor and a CCD commercial digital fixed camera. Results show an excellent performance.
\end{abstract}

\section{INTRODUCTION}

Robot tasks that involve joint encoders, force sensors, CCD cameras, proximity sensors, haptic interfaces, and tactual devices pose a challenging problem in robotics due to the the multisensor nature of the problem and the nonlinear dynamics of the robot. However, it is well known that multisensor-based robot control approaches may offer a solution to very important and relevant, but complex, problems in robotics. In order to achieve sensor fusionbased controller, a careful analysis of the dynamics, sensors behavior, and tasks are required. Furthermore, since physical parameters are in practice uncertain, robustness to parametric uncertainties are an integral part of the control problem. One example of such tasks, is the force-position control of a robot using visual information, where force, joint encoders and visual sensor have to be fused. In this paper, we focus in the paradigm: "design a controller that ensures tracking of image-based trajectories of constrained robots subject to uncertainties on camera, robot, contact and joint dynamic friction parameters". This task is very relevant in many robotic applications. Therefore, for any practical impact, robot, camera and friction uncertainties must be considered.
This problem has been elusive because it is not evident how to deal with vision and force signals, despite lot of the availability of schemes of vision or force. In this paper, this problem is studied offering a formal solution, and its experimental evaluation.

\section{Motivation AND CONTRIBUtion}

\section{A. Motivation}

Image-based visual servoing schemes of robot manipulators for free motion have been proposed recently, which guarantee tracking, including the dynamic model in the stability analysis. The task under study is that the robot end effector tracks a visual trajectory along the surface of an object, and at the same time, control the applied force exerted in the surface by the end-effector, taking into account that when two rigid surfaces are in contact, friction is presented between them, and must be considered for any practical application, see Fig. 1. However, for constrained robot, there remains important open problems, essentially because, from the theoretical viewpoint, it involves redundant sensors, thus it is not evident how to handle sensor fusion in a complex nonlinear dynamical system. From the experimental viewpoint, besides that exhibits a multirate system due to the slow latency of the camera, in comparison to the latency of the encoder and force sensors, also, presents dynamic friction at the joints and at least viscous contact friction. Therefore, a theoretical constrained visual servoing scheme must be accompanied with its experimental validation. ${ }^{1}$ In this paper, a new scheme and its real time performance, are proposed.

\section{B. Friction}

Friction is quite important to compensate because it is a dominant dynamical force in slow and velocity reversal regimes which are typical on visual servoing controls and contact tasks. Nevertheless dynamic joint and contact friction

\footnotetext{
${ }^{1}$ Two points arise here: Notice that the static state of the camera is not a generalized coordinate of the dynamical system, thus its slow latency is not an issue; however, the generalized coordinates are required to be sampled very fast, in comparison to its natural frequency. In this way, the system can be treated in the time domain, even though its implementation is carried out in the digital/discrete domain.
} 


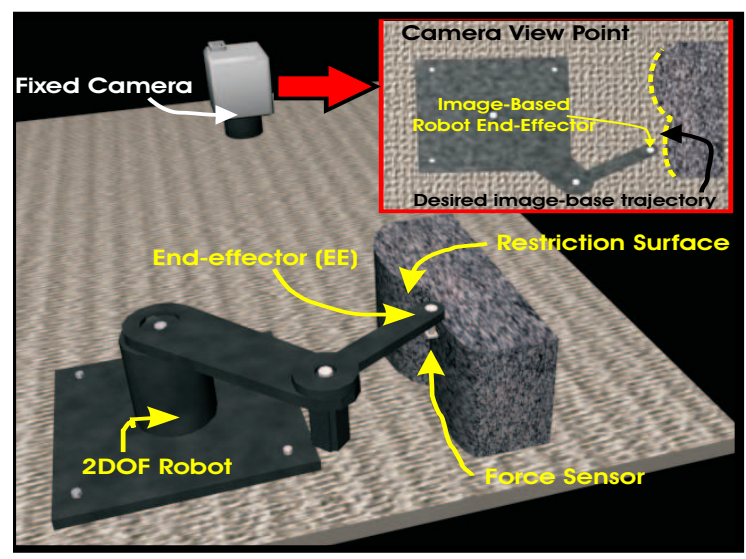

Fig. 1. Robot Force-Vision Experimental System.

is usually neglected in motion control, and unfortunately, it is not the exception in visual servoing literature. Therefore, we consider the LuGre model ${ }^{2}$, which reproduces pre-sliding regime at very small displacements and hard nonlinearities for slow motion and velocity reversals. The problem complicates because, although dynamic joint friction depends on joint coordinates, in image-based control, contact friction depends on image coordinates, therefore, interestingly, it is required a visual friction compensator.

\section{Contribution}

A sliding mode adaptive controller driven by constrained image errors is proposed to solve by rst time the problem possed above. The underlying reason that allows to obtain this result is that a new image-based error manifold is introduced to produce a visual-based orthogonalized principle. Thus, similar results to the case of nonvisualbased orthogonalized principle are obtained. The closed-loop system guarantees exponential tracking of position and force trajectories subject to parametric uncertainties. This scheme delivers a smooth controller and presents formal stability proofs. Moreover, its experimental validation is presented

We further extend our proposal to include visual compensation of dynamic friction. Surprisingly, the control structure is quite simple, in contrast, the proof is rather involved, though straightforward. The simplicity of the controller enhances its practical applications since the desired task is designed in image space, i.e., the user de nes the desired task right from the image that sees ${ }^{3}$, see Fig. 1, wherein the xed camera supplies a perspective of the desired task.

\section{BACKGROUND}

Hybrid vision/force control approaches have been reported [1] [4], and none of them shows robustness to uncertainties, on robot parameters and camera parameters. In a different path, the authors Xiao et al. [5], present an interesting scheme

\footnotetext{
${ }^{2}$ This dynamic friction is responsible for limit cycles.

${ }^{3}$ Provided that the xed position of the camera is set to covers the reachable space of the robot, in this way a task free of singularities is ensured.
}

of hybrid vision force control in an uncalibrated environment, but their approach does not deal with uncertainties of robot parameters, and exhibits a very complex control law.

With respect to force control, Arimoto solved by rst time the simultaneous control of position and force using the full nonlinear dynamics subject to parametric uncertainties without coordinate partitioning. This was possible through judicious design of extended error, that is based on the orthogonalization principle [6]. Afterwards, several schemes have been proposed based on the orthogonalization principle, however these schemes have not been extended or combined beyond constrained robots.

\section{NONLINEAR ROBOT DYNAMICS}

\section{A. Constrained robot dynamics}

The constrained robot dynamics arises when its end effector is in contact to in nitely rigid surface. Considering the generalized joint position $q \in \Re^{2}$ and velocity coordinates $\dot{q} \in \Re^{2}$, this 2-DOF constrained robot system can be modeled as follows: [6]

$$
\begin{aligned}
H(q) \ddot{q}+C(q, \dot{q}) \dot{q}+g(q) & =\tau+J_{\varphi+}^{T}(q) \lambda-F(\dot{q}, \dot{z}, z) \\
& -J^{T}(q) B_{t} J(q) \dot{q} \\
\varphi(q) & =0
\end{aligned}
$$

where $H(q) \in \Re^{2 \times 2}$ stands for the robot inertia matrix; $C(q, \dot{q}) \dot{q} \in \Re^{2}$ stands for the vector of centripetal and Coriolis torques; $g(q) \in \Re^{2}$ is the vector of gravitational torques, $F(\dot{q}, \dot{z}, z)$ is the dynamic friction ${ }^{4}, B_{t} \in R_{+}^{2 \times 2}$, is the viscous friction matrix, possibly not a diagonal matrix, $J^{T}(q) B_{t} J(q) \dot{q}$ represents the tangential viscous friction at the contact point, $J_{\varphi+}(q)=\frac{J_{\varphi}}{J_{\varphi} J_{\varphi}^{T}}$ is the constrained normalized jacobian of the the kinematic constraint $\varphi(q)=0$, or rigid surface with continuous gradient, $\lambda$ is the constrained lagrangian, or contact force and $\tau \in \Re^{2}$ stands for the vector of input torque control.

Adding and subtracting to (1) the linear parametrization $H(q) \ddot{q}_{r}+C(q, \dot{q}) \dot{q}_{r}+g(q)+J^{T}(q) B_{t} J(q) \dot{q}=y_{r} \theta_{b}$, where the known regressor $y_{r} \in \Re^{2 \times p}$ and the unknown constant vector $\theta_{b} \in \Re^{p}$, produces the open loop error equation,

$$
\begin{gathered}
H(q) \dot{S}_{q}=-C(q, \dot{q}) S_{q}-J^{T}(q) B_{t} J(q) S_{q}+ \\
\tau+J_{\varphi}^{T}(q) \lambda-y_{r} \theta_{b}
\end{gathered}
$$

with joint error surface $S_{q}$ is de ned as:

$$
S_{q}=\dot{q}-\dot{q}_{r}
$$

where $\dot{q}_{r}$ stands for the nominal reference of joint velocities, not yet de ned.

\section{CAMERA MOdel}

The robot direct kinematics is de ned as:

$$
x_{b}=f(q)
$$

${ }^{4}$ For a clear exposition, rstly, $F(\dot{q}, \dot{z}, z)$ will be considered zero, however in Section IX it will be treated. 
where $x_{b} \in \Re^{2}$ is the position of robot end effector. Then, the differential kinematics, which relates velocities in cartesian space $\dot{x}_{b} \in \Re^{2}$ to joint space velocities $\dot{q} \in \Re^{2}$, is de ned as follows:

$$
\dot{x}_{b}=J(q) \dot{q}
$$

Now, the visual position $x_{s} \in \Re^{2}$ of robot end effector is [7]:

$$
x_{s}=\alpha_{h} R(\theta) x_{b}+\beta_{s}
$$

where $\alpha_{h}=\alpha_{s} h$, is the scale factor ${ }^{5}$, and $R(\theta) \in S O(2)$, $\beta_{s} \in \Re^{2}$ that depends on intrinsic and extrinsic parameters of camera ${ }^{6}$. The differential camera model is then

$$
\dot{x}_{s}=\alpha_{h} R(\theta) \dot{x}_{b}
$$

where $\dot{x}_{s} \in \Re^{2}$ determines the visual robot end effector velocity. Using equation $(5) \sim(7)$, equation (8) becomes

$$
\dot{x}_{s}=\alpha_{h} R(\theta) J(q) \dot{q}
$$

Thus, the inverse differential kinematics for robot manipulator in terms of visual velocities ${ }^{7}$ becomes

$$
\dot{q}=J(q)^{-1} R(\theta)^{-1} \alpha_{h}^{-1} \dot{x}_{s} \Rightarrow \dot{q}=J_{R i n v} \dot{x}_{s}
$$

This relation is useful to design the nominal reference of joint velocities $\dot{q}_{r}$ in the following section.

\section{VisUal ORThogonalization PRINCIPLE}

Since $\varphi(q)=0 \forall t$, then its time derivative yields $\frac{d}{d t} \varphi(q)=\frac{\partial \varphi(q)}{\partial q} \frac{d q}{d t} \equiv J_{\varphi}(q) \dot{q}=0$. This means that $J_{\varphi}(q)$ is orthogonal to $\dot{q}$. That is, $\dot{q}$ belongs to the orthogonal projection matrix $Q=I-J_{\varphi+}^{T} J_{\varphi}$ of $J_{\varphi}(q)$ [6]. As we can see, $Q$ spans the tangent plane at the contact point, therefore, $J_{\varphi}$ and $Q$ are orthogonal complements. In other words, if the robot end effector is in contact with the constraint surface, then $Q \dot{q}=\dot{q} \rightarrow Q Q \dot{q}=Q \dot{q} \equiv \dot{q} \Rightarrow Q J_{\varphi}^{T}=0$. These properties are fundamental to set the visual orthogonalization principle as follows. Firstly consider $\dot{q}_{r}$ in terms of orthogonal nominal references of velocity $\dot{q}_{s}$ and force $\dot{q}_{f}$, as follows

$$
\dot{q}_{r}=\dot{q}_{s}+\dot{q}_{f}
$$

Notice that, engagingly, an image based servo visual force control without computing inverse kinematics is designed ${ }^{8}$, then nominal reference $\dot{q}_{r}$ must be designed in terms of nominal visual reference and nominal force reference as follows

$$
\dot{q}_{r}=Q J_{R i n v} \dot{x}_{r}+\beta J_{\varphi}^{T} \dot{q}_{r f}
$$

Using (10) consider now the next nominal visual reference of velocities

$\dot{x}_{r}=\dot{x}_{s d}-\alpha \Delta x_{s}+S_{s d}-\gamma_{s 1} \int_{t_{0}}^{t} S_{s \delta}-\gamma_{s 2} \int_{t_{0}}^{t} \operatorname{sign}\left(S_{s \delta}\right)$

\footnotetext{
${ }^{5}$ Without loss of generality, $\alpha$ can be considered as a scalar matrix $2 \times 2$.

${ }^{6}$ Focal distance, depth of eld, translation of camera center to image center, distance between optical axe to the robot base.

${ }^{7}$ With $J_{\text {Rinv }} \in \Re^{n \times n}$ whose entries are functions of robot and camera parameters.

${ }^{8}$ to eliminate the inverse kinematics calculus and to reduce the control law computational cost. This is also one byproduct of this scheme.
}

where $\dot{x}_{s d}$ stands for desired visual velocity trajectory, and $\Delta x_{s}=x_{s}-x_{s d}$ is the visual position error. for $S_{s \delta}=$ $S_{s}-S_{s d} \equiv\left(\Delta \dot{x}_{s}+\alpha \Delta x_{s}\right)-S_{s}\left(t_{0}\right) e^{-\kappa_{s} t}$, where $\Delta \dot{x}_{s}=$ $\dot{x}_{s}-\dot{x}_{s d}$ de nes visual velocity error, $\kappa_{s}>0$ and $\gamma_{s_{i}}=\gamma_{s_{i}}^{T} \in$ $\Re_{+}^{n \times n}, i=1,2$.

Now, let consider the nominal force reference as

$$
\dot{q}_{r f}=\Delta F-S_{d F}+\gamma_{F 1} \int_{t_{0}}^{t} S_{F \delta}+\gamma_{F 2} \int_{t_{0}}^{t} \operatorname{sign}\left(S_{F \delta}\right)
$$

for $S_{F \delta}=S_{F}-S_{F d} \equiv \Delta F-S_{F}\left(t_{0}\right) e^{-\kappa_{F} t}$, where $\Delta F=$ $\int_{t_{0}}^{t} \Delta \lambda(\zeta) d \zeta, \Delta \lambda=\lambda-\lambda_{d}, \lambda_{d}$ is the desired contact force, $\kappa_{F}>0$, and $\gamma_{F_{i}}=\gamma_{F_{i}} \in \Re_{+}^{n \times n}, i=1,2$.

Using equations (12), (13), (14) and (10) into (4), the visual orthogonalized joint error surface is:

$$
\begin{aligned}
S_{q} & =\dot{q}-\dot{q}_{r} \equiv Q \dot{q}-\dot{q}_{r} \\
& =Q J_{R i n v} \dot{x}_{s}-Q J_{R i n v} \dot{x}_{r}-\beta J_{\varphi}^{T} \dot{q}_{r f} \\
& =Q J_{R i n v} S_{v s}-\beta J_{\varphi}^{T} S_{v F}
\end{aligned}
$$

with

$$
\begin{aligned}
S_{v s} & =S_{s \delta}+\gamma_{s 1} \int_{t_{0}}^{t} S_{s \delta}+\gamma_{s 2} \int_{t_{0}}^{t} \operatorname{sign}\left(S_{s \delta}\right) \\
S_{v F} & =S_{F \delta}+\gamma_{F 1} \int_{t_{0}}^{t} S_{F \delta}+\gamma_{F 2} \int_{t_{0}}^{t} \operatorname{sign}\left(S_{F \delta}\right)
\end{aligned}
$$

where $S_{v s}$ stands for the visual manifold and $S_{v F}$ stands for the force manifold.

Notice that $S_{q}$ is composed of two orthogonal complements $Q J_{\text {Rinv }} S_{v s}$ depending on image coordinate error, and $\beta J_{\varphi}^{T} S_{v F}$ depending of integral of contact force errors. Thus, tracking errors $\Delta x_{s}$ and $\Delta F$ can be controlled independently, since they are mapped to orthogonal complements.

Remark 1. The above de nition assumes exact knowledge of $J_{\text {Rinv }}$. However, in practice, it stands as a very restricted assumption. Therefore, we need to design a uncertain manifold $S_{q}$ taking into consideration the uncertainty of $J_{\text {Rinv }}$. To this end, consider

$$
\widehat{\dot{q}}_{r}=Q \widehat{J_{R i n v}} \dot{x}_{r}+\beta J_{\varphi}^{T} \dot{q}_{r f}
$$

with $\widehat{J_{\text {Rinv }}}$ an estimated of $J_{R i n v}$, such that rank $\widehat{J^{-1}(q)}$ and $\widehat{R_{\alpha}^{-1}(\theta)}$ are full rank $\forall q \in \Omega$, where the robot workspace free of singularities is de ned by $\Omega=$ $\left\{q \mid \operatorname{rank}(J(q))=n, \forall q \in \Re^{n}\right\}$, and $\forall \theta \in \Re$. Thus, substituting (16) into (4), we have the uncalibrated joint error surface

$$
\begin{aligned}
\hat{S}_{q} & =\dot{q}-\widehat{\dot{q}}_{r} \\
& =Q J_{R i n v} \dot{x}_{s}-Q \widehat{J_{R i n v}} \dot{x}_{r}-\beta J_{\varphi}^{T} \dot{q}_{r f}
\end{aligned}
$$

where $\hat{S}_{q}$ is available because $\dot{q}$ and $\widehat{\dot{q}}_{r}$ are available. Adding and subtracting $Q J_{R i n v} \dot{x}_{r}$ to (17) we obtain:

$$
\begin{aligned}
\hat{S}_{q} & =Q J_{R i n v} S_{v s}-\beta J_{\varphi}^{T} S_{v F}-Q \Delta J_{R i n v} \dot{x}_{r} \\
& =S_{q}-Q \Delta J_{R i n v} \dot{x}_{r}
\end{aligned}
$$

where $\Delta J_{\text {Rinv }}=\widehat{J_{R i n v}}-J_{R i n v}$. 


\section{OPEN LOOP ERROR EQUATION}

Using (16), the uncertain parametrization is: $H(q) \widehat{\ddot{q}}_{r}+$ $C(q, \dot{q}) \widehat{\dot{q}}_{r}+g(q)+J^{T}(q) B_{t} J(q) \dot{q}=y_{r} \hat{\theta}_{b}$, where $\widehat{\ddot{q}}_{r}=$ $f\left(\ddot{x}_{r}, \ddot{q}_{f r}\right)$, with

$$
\begin{aligned}
\ddot{x}_{r} & =\ddot{x}_{s d}-\alpha \Delta \dot{x}_{s}+\dot{S}_{s d}-\gamma_{s 1} S_{s \delta}-\gamma_{s 2} \operatorname{sign}\left(S_{s \delta}\right) \\
\ddot{q}_{r f} & =\Delta \dot{F}-\dot{S}_{d F}+\gamma_{F 1} S_{F \delta}+\gamma_{F 2} \operatorname{sign}\left(S_{F \delta}\right)
\end{aligned}
$$

which introduces discontinuous terms. To avoid introducing high frequency discontinuous signals, add and subtract $\tanh \left(v_{s} S_{s \delta}\right)$ and $\tanh \left(v_{f} S_{F \delta}\right), v_{f}, v_{s}>0$, to $\ddot{q}_{r}$ to separate continuous and discontinuous signals as follows

$$
\widehat{\ddot{q}}_{r}=\widehat{\ddot{q}}_{r c o n t}+Q \gamma_{s 2} z_{s}-\beta J_{\varphi}^{T} \gamma_{F 2} z_{f}
$$

with $z_{s}=\tanh \left(v_{s} S_{s \delta}\right)-\operatorname{sign}\left(S_{s \delta}\right)$ and $z_{f}=\tanh \left(v_{f} S_{F \delta}\right)-\operatorname{sign}\left(S_{F \delta}\right)$. Thus $y_{\text {cont }}=$ $y_{r}\left(q, \dot{q}, \widehat{\dot{q}}_{r}, \widehat{\ddot{q}}_{r c o n t}\right)$ is continuous since $\left(\widehat{\dot{q}}_{r}, \widehat{\ddot{q}}_{r c o n t}\right) \in \mathcal{C}^{1}$, where

$$
\begin{aligned}
\widehat{\ddot{q}}_{\text {rcont }}= & Q \widehat{Q J_{\text {Rinv }}} \ddot{x}_{r c o n t}+\dot{Q} \widehat{J_{\text {Rinv }}} \dot{x}_{r c o n t}+Q \widehat{\dot{J}_{\text {Rinv }}} \dot{x}_{r c o n t} \\
& \beta J_{\varphi}^{T} \ddot{q}_{r f c o n t}+\beta \dot{J}_{\varphi}^{T} \dot{q}_{r f c o n t}
\end{aligned}
$$

with

$$
\begin{aligned}
\ddot{x}_{r c o n t} & =\ddot{x}_{s d}-\alpha \Delta \dot{x}_{s}+\dot{S}_{s d}-\gamma_{s 1} S_{s \delta}-\gamma_{s 2} \tanh \left(v_{s} S_{s \delta}\right) \\
\ddot{q}_{r f c o n t} & =\Delta \dot{F}-\dot{S}_{d F}+\gamma_{F 1} S_{F \delta}+\gamma_{F 2} \tanh \left(v_{f} S_{F \delta}\right)
\end{aligned}
$$

Therefore the uncertain parametrization becomes

$$
\begin{aligned}
& H(q) \hat{\hat{q}_{r}}+C(q, \dot{q}) \hat{\hat{q}_{r}}+g(q)+J^{T}(q) B_{t} J(q) \hat{\dot{q}_{r}}= \\
& y_{\text {cont }} \hat{\theta}_{b}+H\left(Q \gamma_{s 2} z_{s}-\beta J_{\varphi}^{T} \gamma_{F 2} z_{f}\right)
\end{aligned}
$$

Adding and subtracting (25) to (1), we obtain nally the open loop error in function of $\left(q, \dot{q}, \hat{\dot{q}}_{r}, \hat{\ddot{q}}_{\text {rcont }}\right)$ as follows:

$$
\begin{aligned}
H(q) \widehat{\dot{S}_{q}}= & \tau-C(q, \dot{q}) \widehat{S_{q}}+J_{\varphi+}^{T}(q) \lambda- \\
& y_{\text {cont }} \hat{\theta}_{b}-J^{T}(q) B_{t} J(q) \widehat{S_{q}}+ \\
& H\left(Q \gamma_{s 2} z_{s}-\beta J_{\varphi}^{T} \gamma_{F 2} z_{f}\right)
\end{aligned}
$$

Now we are ready to present the main result.

\section{Control Design}

Theorem 1 Assume that initial conditions and desired trajectories belong to $\Omega$, and consider the robot dynamics (1) in closed loop with the following visual adaptive forceposition control law

$$
\begin{aligned}
\tau= & -K_{d} \hat{S}_{q}+y_{\text {cont }} \hat{\theta}_{b}+J_{\varphi+}^{T}(q)\left[-\lambda_{d}+\eta \Delta F\right]+ \\
& \beta J_{\varphi}^{T}(q) *\left[\tanh \left(v_{F} S_{F \delta}\right)+\eta \int_{t_{o}}^{t} \operatorname{sgn}\left(S_{F \delta}\right)\right] \\
\dot{\hat{\theta}}_{b}=- & -\Gamma y_{\text {cont }}^{T} \hat{S}_{q}
\end{aligned}
$$

where $\gamma_{s 1}, \gamma_{F 1}>0, \quad \Gamma \in \Re_{+}^{p \times p_{+}}, K_{d} \in$ $\Re_{+}^{2 \times 2}, \beta, \eta>0$. If $K_{d}$ is large enough and error of initial conditions are small enough, and if $\gamma_{s 2} \geq\left\|\frac{d}{d t}\left\{R_{\alpha}(\theta) J(q)\left[\hat{S}_{q}+\left(\Delta J_{R i n v}\right) \dot{x}_{r}\right]\right\}\right\|, \gamma_{F 2} \geq$ $\left\|\frac{d}{d t}\left[\left(J_{\varphi} J_{\varphi}^{T}(q)\right)^{-1} J_{\varphi} \hat{S}_{q}\right]\right\|$, then exponential convergence of visual and force tracking errors is guaranteed.

Proof: The proof can be found in the appendix.

Remark 2. Apparently there is problem with $J(q(t))^{-1}$. However, we have proved that $J(q(t))$ is not singular for all time, because $q(t) \rightarrow q_{d}(t)$ exponentially, without overshoot, with desired trajectories belonging to robot workspace $\Omega$, thus $J(q(t)) \rightarrow J\left(q_{d}(t)\right)$ within $\Omega$ and $J(q(t))^{-1}$ is wellposed $\forall t$.

\section{DYNAMIC FRICTION COMPENSATION}

Now let us consider the dynamic friction into the model, which represent a very realistic behavior when the robot is moving along a rigid surface, in particular, driven by visual servoing. In this case, the following LuGre [8] dynamic friction model is very suitable to de ne the joint friction

$$
\begin{aligned}
F(\dot{q}, \dot{z}, z) & =\sigma_{0} z+\sigma_{1} \dot{z}+\sigma_{2} \dot{q} \\
\dot{z} & =-\sigma_{0} h(\dot{q}) z+\dot{q} \\
h(\dot{q}) & =\frac{|\dot{q}|}{\alpha_{0}+\alpha_{1} \exp p^{-\left(\dot{q} / \dot{q}_{s}\right)^{2}}}
\end{aligned}
$$

where matrix parameters $\sigma_{1}, \sigma_{2}, \sigma_{3} \in \Re^{n \times n}$ are diagonal de nite matrices, the state $z \in R^{2}$ stands for the position of the bristles, $\alpha_{0}, \alpha_{1}>0$, and $\dot{q}_{s}>0$. This model involve a very complex dynamics around the trivial equilibrium, for bidirectional motion, and for very small displacements, the forces that comes out from this model makes impossible to reach the origin due to limit cycles induced and the potentially unstable behavior. Substituting (29) into (1) yields

$$
\begin{aligned}
& H(q) \ddot{q}+C(q, \dot{q}) \dot{q}+J(q)^{T} B_{t} J(q) \dot{q}+\sigma_{12} \dot{q}+ \\
& g(q)+\sigma_{0} z-\sigma_{01} h(\dot{q}) z=\tau+J_{\varphi}^{T}(q) \lambda
\end{aligned}
$$

where $\sigma_{01}=\sigma_{0} \sigma_{1}$ and $\sigma_{12}=\sigma_{1}+\sigma_{2}$. Now, we need to organize the parametrization in terms of two regressors: $H(q) \hat{\ddot{q}}_{\text {cont }}+\left(C(q, \dot{q})+\widehat{J(q)^{T}} B_{t} \widehat{J(q)}+\sigma_{12}\right) \hat{\dot{q}}_{r}+$ $g(q)=Y_{\text {cont }} \hat{\Theta}_{b}$ and the virtual continuous regressor $\frac{\sigma_{01} \alpha_{01}}{\alpha_{0}}|\dot{q}| \tanh \left(\xi_{f} S_{q}\right)+\sigma_{0} \alpha_{01} \tanh \left(\xi_{f} S_{q}\right)=Y_{f} \Theta_{f}$, where $\alpha_{01}=\alpha_{0}+\alpha_{1}, \tanh (q)$ is the continuous hyperbolic tangent function, and $\xi_{f}>0$. Now, If we add and subtract the above regressors to (30) yields the following open-loop error dynamics with dynamic friction

$$
\begin{gathered}
H(q) \hat{\dot{S}}_{q}=-\left(C(q, \dot{q})+J(q)^{T} B_{t} J(q) \hat{S}_{q}+\sigma_{12}\right) \hat{S}_{q}+ \\
\tau-\mathcal{F}-Y \hat{\Theta}+J_{\varphi}^{T}(q) \lambda-\sigma_{0} z+\sigma_{01} h(\dot{q}) z \\
+H\left(Q \gamma_{s 2} z_{s}-\beta J_{\varphi}^{T} \gamma_{F 2} z_{f}\right)
\end{gathered}
$$

where $\mathcal{F}=\sigma_{0} z+\sigma_{0}+\sigma_{0} \alpha_{0}{ }^{-1} \sigma_{1}|\dot{q}|-\sigma_{0} \sigma_{1}|\dot{q}| z \alpha_{e}, \sigma_{x}=$ $\alpha_{01} \tanh \left(\lambda_{f} S_{q}\right), \alpha_{e}=\left(\alpha_{0}+\alpha_{1} \exp ^{-\left(\dot{q} / \dot{q}_{b}\right)^{2}}\right)^{-1}, Y=$ $\left[Y_{\text {cont }}, Y_{f}\right]$, and $\hat{\Theta}=\left[\hat{\Theta}_{b}^{T}, \Theta_{f}^{T}\right]^{T}$. Finally, consider the following control law

$$
\begin{aligned}
\tau & =-K_{d} \hat{S}_{q}+Y \hat{\Theta}+J_{\varphi}^{T}(q)\left[-\lambda_{d}+\eta \Delta F\right] \\
& +\beta J_{\varphi}^{T}(q)\left[\tanh \left(\mu S_{q F}\right)+\eta \int \operatorname{sgn}\left(s_{F \delta}\right)\right] \\
\dot{\hat{\Theta}} & =-\Gamma Y^{T} \hat{S}_{q}
\end{aligned}
$$


where $\Gamma \in R_{+}^{p \times p}, K_{d} \in R_{+}^{n \times n}, \beta, \eta>0$. We now have the following result.

Theorem 2 Assume that initial conditions and desired trajectories belong to $\Omega$, and consider the controller (32)-(33). If $K_{d}$ is large enough and a error of initial conditions are small enough, and if $\gamma_{s 2} \geq\left\|\frac{d}{d t}\left\{R_{\alpha}(\theta) J(q)\left[\hat{S}_{q}+\Delta J_{R i n v} \dot{x}_{r}\right]\right\}\right\|, \quad \gamma_{F 2} \geq$ $\left\|\frac{d}{d t}\left[\left(-\beta J_{\varphi} J_{\varphi}^{T}(q)\right)^{-1} J_{\varphi} \hat{S}_{q}\right]\right\|$, then exponential convergence of visual and force tracking errors is guaranteed.

Proof: The proof can be found in the appendix.

Remark 3. Important properties of this control scheme have to be highlighted: Is an Image-based dynamical control scheme for constrained robots that fuses visual, encoder and force signals. This control law presents, for first time in literature, compensation of dynamic friction by means of visual feedback.

\section{EXPERIMENTAL STATION}

Robot parameters and constant gains used in the experiments are shown in Table I, and an image of the experimental setup is depicted in Fig. 2, meanwhile, camera parameters are: $\alpha_{s}=79729.0$ pixels $/ \mathrm{m}, h=-0.006 \mathrm{~m}$, $\beta_{s}=O_{s i}+\alpha_{h} R(\theta) O_{v b}, O_{v b}=[0.257,0.017] m, O s i=$ $[347,266]$ pixels, $\theta=\frac{\pi}{2}$ rad. The robot and camera parametric uncertainty is $10 \%$ for each parameter, i.e., $l_{1}, l_{2}, \alpha_{s}$, etc. The robot is initialized with a high gain PD since the vector $\hat{\Theta}\left(t_{0}\right)$ equals zero. Thus, friction parameters are not required to be known, because these ones are included in $\hat{\Theta}$ (see eq.(31), and the subsequent de nition of $\hat{\Theta}$ ).

\section{TABLE I}

PARAMETERS (Par) OF THE ROBOT, AND FEEDbACK GAINS.

\begin{tabular}{|c|c|c|c|c|c|}
\hline Par & Value & Gain & Value & Gain & Value \\
\hline$m_{1}$ & $6.72 \mathrm{Kg}$ & $K_{d}$ & $\operatorname{diag}(20,1.65)$ & $\beta$ & 0.1 \\
\hline$m_{2}$ & $2.03 \mathrm{Kg}$ & $\gamma_{s 1,2}$ & $\operatorname{diag}(0.1)$ & $\gamma_{F 1,2}$ & 0.01 \\
\hline$l_{1}$ & $0.4 \mathrm{~m}$ & $\alpha$ & $\operatorname{diag}(5.0)$ & $\eta$ & 0.3 \\
\hline$l_{2}$ & $0.3 \mathrm{~m}$ & $\Gamma$ & $\operatorname{diag}(0.01)$ & $\kappa_{1}, \kappa_{2}$ & 20 \\
\hline
\end{tabular}

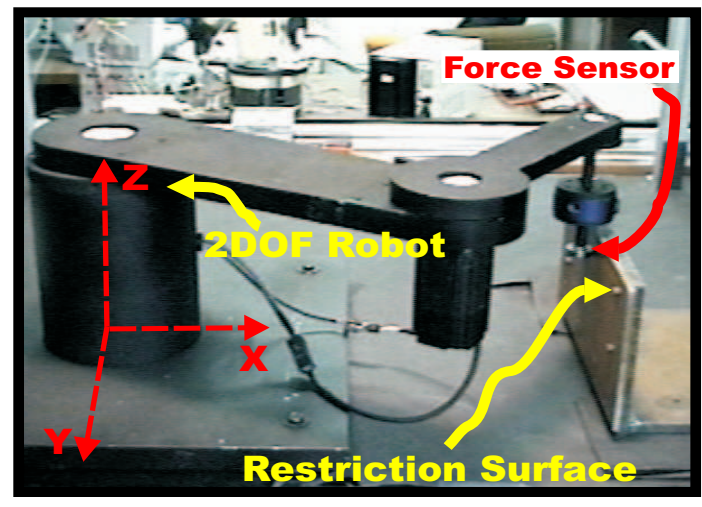

Fig. 2. Experimental setup.

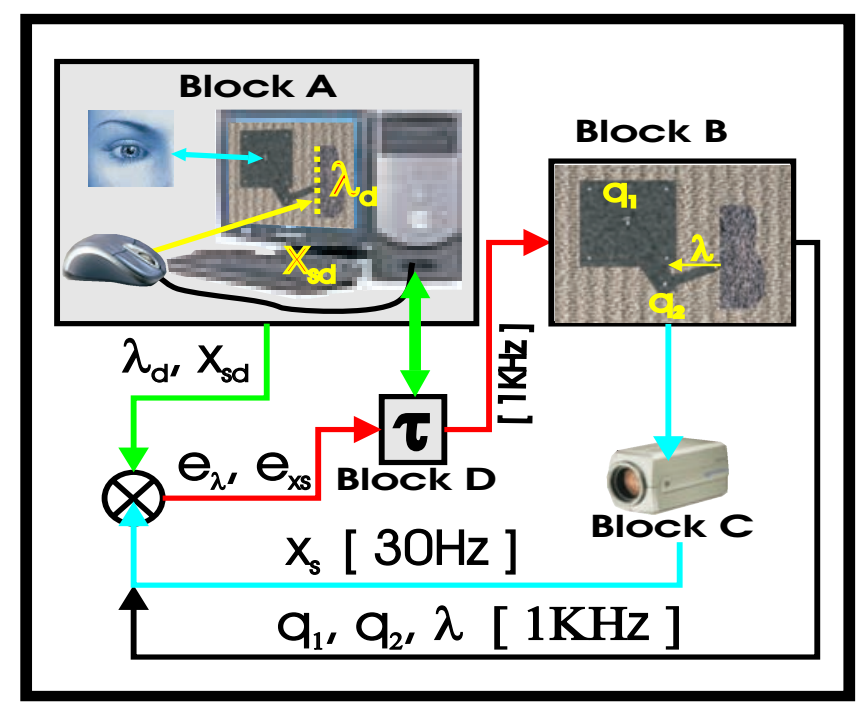

Fig. 3. Fast closed loop of dynamic state vs slow static visual transformation.

\section{A. The Hardware, Software and Firmware}

Fig. 3 shows the experimental system con guration. Block A is composed by a $2.2 \mathrm{GHz}$ personal computer, running over Linux-RTAI OS. This, implements two real-time parallel processes. The rst one, sets communication, at a sample rate of $30 \mathrm{~Hz}$, with the Block C, SONY VFW-V500 camera via IEEE1394 protocol and controls the image processing in order to acquire the robot end-effector position in image space. The second process, Block $\mathbf{D}$, runs with a sampling rate of $1 \mathrm{KHz}$, reads the force sensor and encoders signals computing the torque output for Block B, where the directdrive Yaskawa AC servomotors SGM-08A314 and SGM04U3B4L with 2048 pulse encoders are directly coupled to the links of the 2-dof arm. The Block $\mathbf{B}$ is also integrated by two digital drives Yaskawa servopacks (SGD08AS and SGDA-04AS), and a six-axis force-moment sensor 67M25A-I40-200N12 by JR3 Inc., provided with a DSP Based Interface System for PCI bus. The force sensor is mounted to the end effector of the robot with a rigid aluminum probe with a bearing in its tip, as is shown in Fig.2. Communication between processes is done by realtime shared memory allocation. Low level programming in the Block A provides the interface to a Sensoray $626 \mathrm{I} / \mathrm{O}$ card which contains internal quadrature encoder interface, with 14 bit analog resolution outputs and digital $I / O$. The user designs the desired image based trajectory, directly form the image displayed in the Block A, and sets the desired force pro le. Velocity is computed using a dirty Euler numerical differentiation formula ltered with a lowpass second order Butterworth lter, with a cutoff frequency of $20 \mathrm{~Hz}$.

\section{B. The Task}

The robot task is to move its tool-tip along a speci ed image-based trajectory over the steel surface while at the same time exerts a speci ed pro le of force normal to the surface. The robot is initialized with a high gain PD. The 
inertial frame of the whole system is at the base of the robot and the contact surface is at $y=136$ pixel rendering a $X Z$ plane.

The experiment is performed as follows:

1) From $t=0 \mathrm{~s}$ to $t=3 \mathrm{~s}$. The end effector is requested to move, in free motion, i.e, $J_{\varphi}^{T}=0, Q=I^{9}$, from its initial condition until it makes contact with the surface. The end effector lasts 2 more seconds static.

2) From time $t=5 \mathrm{~s}$ to $t=8 \mathrm{~s}$. Once the tool-tip is in contact with the surface, the control force term is switched on and the tool-tip exerts a desired pro le of force normal to the surface ( 0 to $5 \mathrm{~N}$ ) while moving along $\mathrm{X}$ axis from 230 pixels to 299 pixels

3) From $t=8 \mathrm{~s}$ to $t=12 \mathrm{~s}$. The exerted force is incremented from 5 to 7.5 Newtons, while moving along $\mathrm{X}$ axis from 299 pixels to 230 pixels, as can be seen in Fig. 4 and Fig. $5 \& 6$.

\section{Results}

Fig. 9 shows the input torques. It can be observed that there are not saturation problems and the smooth behavior. Fig. 8 depicts the tracking of real toward the desired trajectories in the cartesian plane. The seemingly high frequency is due this tasks requires very precise control, and due to the sensor resolution is limited to 1pixel.

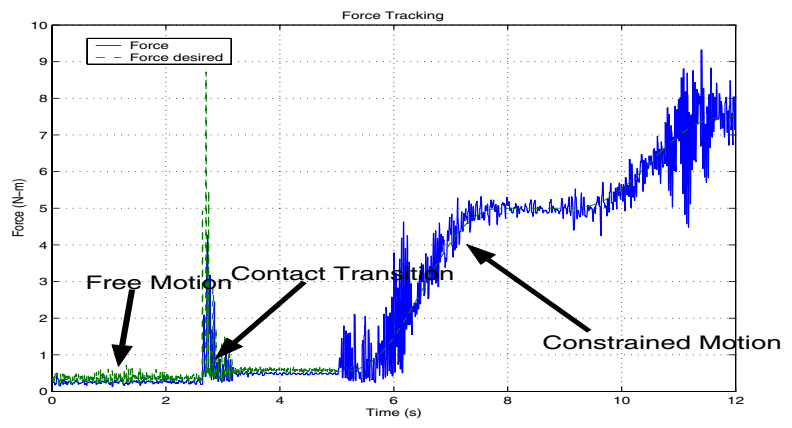

Fig. 4. Force Tracking

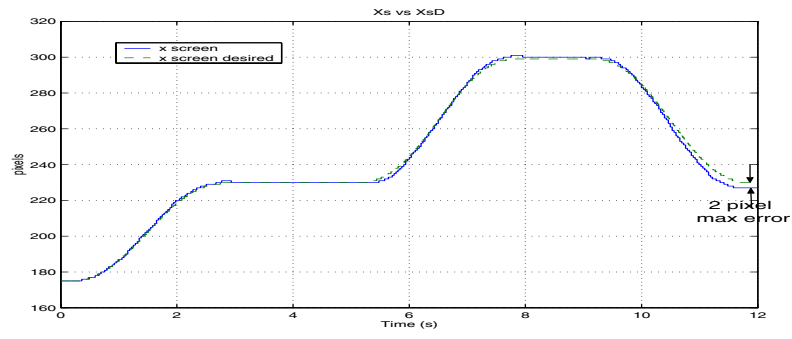

Fig. 5. End effector x position in image space (pixels).

\footnotetext{
${ }^{9}$ It is rather easy to prove that this scheme is stable for unconstrained motion
}

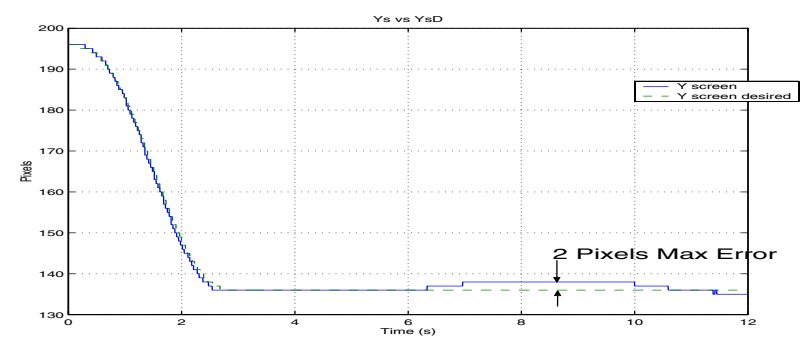

Fig. 6. End effector position in image space (pixels).

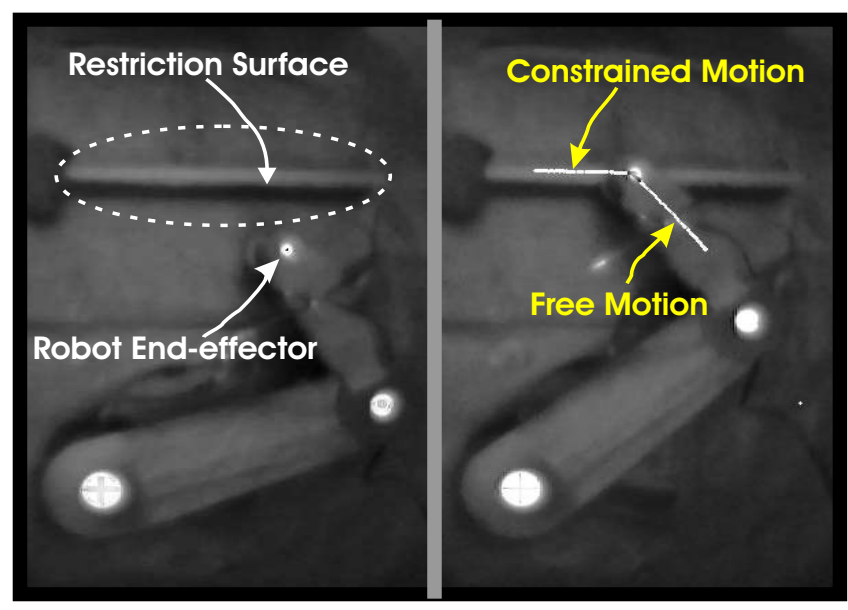

Fig. 7. Experimentation camera view point.

\section{Conclusions}

This paper introduces a novel scheme for adaptive image based visual servoing for force control tasks in constrained dynamical robots. The main feature is the ability to fuse image coordinates into an orthogonal complement of joint velocities, and contact forces. This allows to yield exponential convergence for image-based position-velocity and contact forces even when robot parameters, camera parameters, contact viscous friction and analytical jacobian are considered unknown. Additionally, a compensator of uncertain joint dynamic friction is also presented. Notice that the stability is preserved even when the robot end-effector motion switches from free motion to constrained motion due to its passivity properties, under a set of conditions [13]. Experimental results comply with the theoretical properties.

\section{ACKNOWLEDGEMENTS}

E. Dean and L. Garcia thank CONACYT support under a doctoral scholarship \#158973 and \#158613. V. ParraVega acknowledges support from CONACYT project number 39727-Y.

\section{REFERENCES}

[1] A. Namiki, Y. Nakabo, I. I. M. I., High speed grasping using visual and force feedback. Proceedings of the 1999 IEEE International Conference on Robotics and Automation, Detroit, MI, pages 3195 3200 . 


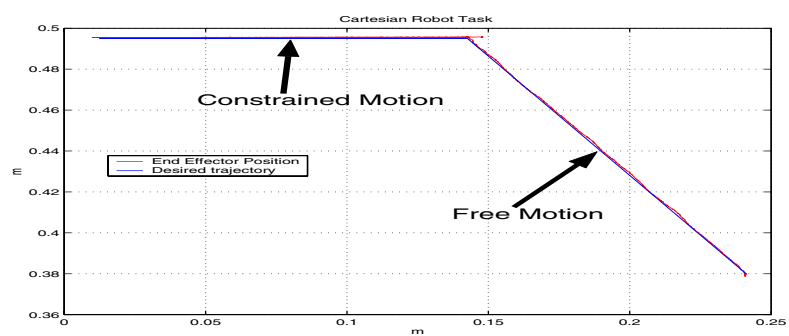

Fig. 8. Cartesian robot task in robot space (m).

[2] B. Nelson, P. (October, 1996), Force and vision resolvability for assimilating disparate sensory feedback, IEEE Trans. on Robot. and Autom.1996, 12(5):714 731.

[3] J. Baeten, W. Verdonck, H. B. J. D. S., Combining force control and visual servoing for planar contour following. Machine Intelligence and Robotic Control. 2000, 2(2):69 75.

[4] S. Jorg, J. Langwald, J. S. G. H. C. N., Flexible robot assambly using a multi-sensory approach, Procc. of the 2000 IEEE Int. Conf. Robot. and Autom., San Francisco, CA, pages 36873694.

[5] Xiao, D., Sensor-hybrid Position/Force control of a robot manipulator in an un calibrated enviroment, IEEE Trans. Control Systm Technology, 2000, 8(4):635 645.

[6] S. Arimoto, Y. H. Liu and T. Naniwa, Model Based Adaptive Hybrid Control for Geometrically Constrained Robot Manipulator, Proc. IEEE Int. Conf. on Rob. and Autom. 1996, pp. 618-623.

[7] S. Hutchinson, G. Hager, P. C., A tutorial on visual servo control, Trans. on Robotics and Automation. 1996. Vol. 6, 651-670

[8] Canudas de Wit, C., Olsson, H., and Astrom, K.J., A New Model for Control of Systems with Friction, IEEE Transactions of Automatic Control, 1995, Vol. 40., No. 3, 419-425.

[9] Panteley, E., Ortega, R., \& Gafvert, M., An Adaptive Friction Compensator for Global Tracking in Robot Manipulators, Systems and Control Letters, 1998, Vol. 33, No. 5, 307 - 313.

[10] V. Parra-Vega, S. Arimoto, Y. L. G. H. P. A., Dynamic sliding PID control for tracking of robot manipulators: Theory and experiments, IEEE Transactions on Robotics and Automation. 2003. Vol. 19., 967976.

[11] Parra-Vega, V., Adaptive compensation of dynamic friction in nite time of 1 DOF mechanical system. American Control Conference, 2001. Proceedings of the 2001 ,Volume: 3 , 25-27 June 2001, pp: 2462 - 2467 vol.3

[12] Garc a-Valdovinos, L. G., Seguimiento Perfecto en Tiempo Finito de Robots Manipuladores Sujetos a Friccion Dinamica, Master Thesis, Dept of EE, Mechatronics Division, CINVESTAV-IPN, Mexico, D.F., 2003.

[13] P. Akella, V. Parra-Vega., Arimoto S. and T. Tanie, Discontinuous Adaptive Control for Robot Manipulators Executing Free and Constrained Tasks , Proc. of the IEEE Robotics and Automation, San Diego (1994), pages 3000-3007.

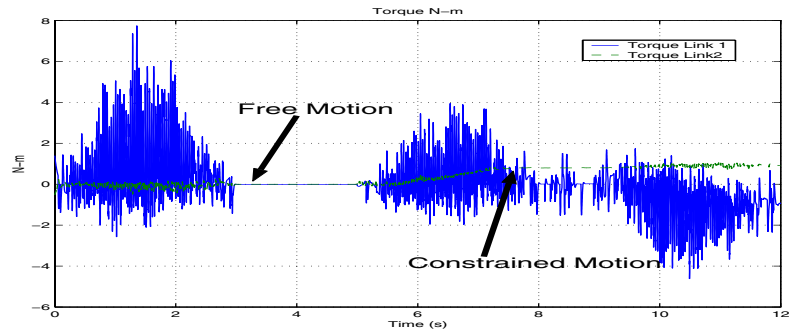

Fig. 9. Smooth input torques.

\section{APPENDIX}

Proof of Theorem1 : The closed loop dynamics between (27) (28) and (26) yields

$$
\begin{aligned}
H(q) \widehat{\hat{S}_{q}=} & -\left\{K_{d}+C(q, \dot{q})\right\} \hat{S}_{q}-y_{\text {cont }} \Delta \theta_{b}+ \\
& J_{\varphi+}^{T}(q)\left[\Delta \lambda+\gamma_{F} \tanh \left(\mu S_{F \delta}\right)\right]+ \\
& \eta J_{\varphi+}^{T}(q)\left[\Delta F+\gamma_{F} \int \operatorname{sgn}\left(S_{F \delta}\right)\right] \\
\Delta \dot{\theta}_{b}= & \Gamma y_{\text {cont }}^{T} \hat{S}_{q}
\end{aligned}
$$

with $\Delta \theta_{b}=\theta_{b}-\hat{\theta}_{b}$. The proof is organized in three parts.

Part I. Boundedness of Closed Loop Trajectories. Consider the time derivative of the following Lyapunov candidate function

$$
V=\frac{1}{2}\left[\hat{S}_{q}^{T} H(q) \hat{S}_{q}+\beta S_{v F}^{T} S_{v F}+\Delta \theta_{b}^{T} \Gamma^{-1} \Delta \theta_{b}\right]
$$

along the solutions of (34)-(35) as

$$
\dot{V} \leq-K_{d}\left\|\hat{S}_{q}\right\|_{2}^{2}-\eta \beta\left\|S_{v F}\right\|+\left\|\hat{S}_{q}\right\| \psi
$$

where $\psi$ is a functional depending on the state and error manifolds, similarly to [10]. Now if $K_{d}$ and $\beta$ are large enough and the initial errors are small enough, we conclude the seminegative de niteness of (37) outside of hyperball $\varepsilon_{0}=\left\{\widehat{S_{q}} \mid \dot{V} \leq 0\right\}$ centered at the origin, such as the following properties of the state of closed loop system arise

$$
\hat{S}_{q}, S_{v F} \in \mathcal{L}_{\infty} \rightarrow\left\|S_{v s}\right\|,\left\|S_{v F}\right\| \in \mathcal{L}_{\infty}
$$

Then, $\left(S_{s \delta}, \int \operatorname{sign}\left(S_{s \delta}\right)\right) \in \mathcal{L}_{\infty}$, and since desired trajectories are $C^{2}$ and feedback gains are bounded, we have that $\left(\widehat{\dot{q}}_{r}, \widehat{\ddot{q}}_{r}\right) \in \mathcal{L}_{\infty}$. The right hand side of (26) shows that $\varepsilon_{1}>0$ exists such that $\left\|\widehat{\hat{S}}_{q}\right\| \leq \varepsilon_{1}$. This result shows only local stability of $\widehat{S_{q}}$ and $\widehat{S_{q}}$. Now we prove that the sliding modes arises.

Rewriting (18) in terms of two orthogonal vectors, we obtain

$$
\hat{S}_{q}=Q\left\{J_{R i n v} S_{v s}-\Delta J_{R i n v} \dot{x}_{r}\right\}-\beta J_{\varphi}^{T}\left\{S_{v F}\right\}
$$

Since $\hat{S}_{q} \in \mathcal{L}_{2}$, and $J_{\text {Rinv }}$ and $Q$ are bounded, then $Q J_{R i n v} S_{v s}$ is bounded and, due to $\varphi(q)$ is smooth and lies in the reachable robot space and $S_{v F} \rightarrow 0$, then $\beta J_{\varphi}^{T} S_{v F} \rightarrow 0$. Now, taking into account that $\widehat{\dot{S}_{q}}$ is bounded, then $\frac{d}{d t} J_{R i n v} Q S_{v s}$ and $\frac{d}{d t} \beta J_{\varphi}^{T} S_{v F}$ are bounded (this is possible because $\dot{J}_{\varphi}^{T}$ is bounded and so $\dot{Q}$ is). All this chains of conclusions proves that there exists constants $\varepsilon_{2}>0$ and $\varepsilon_{3}>0$ such that

$$
\left|\dot{S}_{v s}\right|<\varepsilon_{2},\left|\dot{S}_{v F}\right|<\varepsilon_{3}
$$

Remark 4. Since the continuous tanh(*) is substituted instead of sign $(*)$, upper bounds $\varepsilon_{2}$ and $\varepsilon_{3}$ are greater. To induce the second order sliding mode, and therefore exponential convergence of tracking errors, it suffices to tune $\gamma_{s 2}$ and $\gamma_{F 2}$ to a larger value. Now, we have to prove that for a proper selection of feedback gains $\gamma_{s 1}, \gamma_{s 2}$ and $\gamma_{F 1}$, 
$\gamma_{F 2}$ then trajectories of visual position and force converges to zero. This is possible if we can prove that sliding modes are established in the visual position subspace $Q$ and in the subspace of force $J_{\varphi}^{T}(q)$. Considering that operator $Q J_{\text {Rinv }}$ spans the vector $\hat{S}_{q}$ as the direct sum of its image $i m\left\{Q J_{R i n v}\left(S_{v s}\right)\right\} \equiv S_{v s}^{i m}$ and $i m\left\{\beta J_{\varphi}^{T}\left(S_{v F}\right)\right\} \equiv S_{v F}^{i m}$, see (39), this implies that

$$
\begin{aligned}
\hat{S}_{q} & =Q\left\{J_{R i n v} S_{v s}-\Delta J_{\text {Rinv }} \dot{x}_{r}\right\}-\beta J_{\varphi}^{T}\left\{S_{v F}\right\} \\
& =\left(S_{v s}^{i m}-i m\left\{\Delta J_{R i n v} \dot{x}_{r}\right\}\right)-S_{v F}^{i m}
\end{aligned}
$$

where $S_{v s}^{i m}-i m\left\{\Delta J_{R i n v} \dot{x}_{r}\right\}$ and $S_{v F}^{i m}$ belongs to orthogonal complements, that means $\left\langle S_{v s}^{i m}-i m\left\{\Delta J_{R i n v} \dot{x}_{r}\right\}, S_{v F}^{i m}\right\rangle=$ 0 . That is, we are able to analyze the $S_{v s}^{i m}-i m\left\{\Delta J_{\text {Rinv }} \dot{x}_{r}\right\}$ dynamics independently of $S_{v F}^{i m}$, because $S_{v F}^{i m}$ belongs to the kernel of $Q$. This is veri ed if we multiply (40) by $Q^{T}$, that is

$$
\begin{aligned}
Q^{T} \hat{S}_{q} & =Q^{T} Q\left\{J_{\text {Rinv }} S_{v s}-\Delta J_{\text {Rinv }} \dot{x}_{r}\right\}-\beta Q^{T} J_{\varphi}^{T} S_{v F} \\
& =S_{v s}^{i m}-i m\left\{\Delta J_{\text {Rinv }} \dot{x}_{r}\right\}
\end{aligned}
$$

since $Q$ is idempotent. It is important to notice that if $A x=$ $A y$ for any square nonsingular matrix $A$ and any couple of vectors $x, y$, then $x \equiv y$. Thus, equation (41) means that $\hat{S}_{q}=Q\left\{J_{R i n v} S_{v s}-\Delta J_{R i n v} \dot{x}_{r}\right\}$ is valid within span of $Q$. Now, if we multiply $\hat{S}_{q}$ by $J_{\varphi_{+}}$we obtain

$$
\begin{aligned}
J_{\varphi_{+}} \hat{S}_{q} & =J_{\varphi_{+}} Q\left\{J_{R i n v} S_{v s}-\Delta J_{R i n v} \dot{x}_{r}\right\}-\beta J_{\varphi_{+}} J_{\varphi}^{T}\left\{S_{v F}\right\} \\
& =-\beta S_{v F}
\end{aligned}
$$

\section{Part II: Second Order Sliding Modes.}

Part II.a: Sliding modes for the velocity subspace. According to $Q^{T} \hat{S}_{q}=Q\left\{J_{R i n v} S_{v s}-\Delta J_{R i n v} \dot{x}_{r}\right\}$ then $\hat{S}_{q} \equiv$ $J_{\text {Rinv }} S_{v s}-\Delta J_{R i n v} \dot{x}_{r}$ in the image subspace of $Q$, however notice that $Q$ is not full rank, then this equivalence is valid locally, not globally. In this local neighborhood, if we multiply $\hat{S}_{q}=Q\left\{J_{R i n v} S_{v s}-\Delta J_{R i n v} \dot{x}_{r}\right\}$ by $R_{\alpha}(\theta) J(q)^{10}$, we have

$$
\begin{gathered}
R_{\alpha}(\theta) J(q) \hat{S}_{q}=S_{s \delta}+\gamma_{s 1} \int_{t_{0}}^{t} S_{s \delta}+\gamma_{s 2} \int_{t_{0}}^{t} \operatorname{sign}\left(S_{s \delta}\right)- \\
R_{\alpha}(\theta) J(q)\left\{\Delta J_{\text {Rinv }} \dot{x}_{r}\right\}
\end{gathered}
$$

Taking the time derivative of (43), and multiply it by $S_{s \delta}^{T}$ produces

$$
\begin{aligned}
S_{s \delta}^{T} \dot{S}_{s \delta}= & -\gamma_{s 2} S_{s \delta}^{T} \operatorname{sign}\left(S_{s \delta}\right)-\gamma_{s 1} S_{s \delta}^{T} S_{s \delta}+ \\
& S_{s \delta}^{T} \frac{d}{d t}\left[R_{\alpha}(\theta) J(q)\left(\hat{S}_{q}+\Delta J_{R i n v} \dot{x}_{r}\right)\right] \\
\leq & -\mu_{s}\left|S_{s \delta}\right|-\gamma_{s 1}\left\|S_{s \delta}\right\|^{2}
\end{aligned}
$$

where $\mu_{s}=\gamma_{s 2}-\varepsilon_{4}$, and $\varepsilon_{4}=$ $\frac{d}{d t}\left[R_{\alpha}(\theta) J(q)\left(\hat{S}_{q}+\Delta J_{\text {Rinv }} \dot{x}_{r}\right)\right]$. Thus, we obtain the sliding condition if $\gamma_{s 2}>\varepsilon_{4}$, such as $\mu_{s}>0$ of (44) guarantee the sliding mode at $S_{s \delta}=0$ at $t_{s}=\frac{\left|S_{s \delta}\left(t_{0}\right)\right|}{\mu_{s}}$. However, notice that for any initial condition $S_{s \delta}\left(t_{0}\right)^{\mu_{s}}=0$,

\footnotetext{
${ }^{10}$ Remember the equality: $J_{\text {Rinv }}=J^{-1}(q) R_{\alpha}^{-1}(\theta)$.
}

then $t_{s}=0$, which implies that the sliding mode at $S_{s \delta}(t)=0$ is guaranteed for all time.

Part II.b: Sliding modes for the force subspace. Similarly, if we multiply (42) by $\left(J_{\varphi} J_{\varphi}^{T}(q)\right)^{-1}$, we obtain

$$
\begin{aligned}
\left(J_{\varphi} J_{\varphi}^{T}(q)\right)^{-1} J_{\varphi} \hat{S}_{q} & =-\beta J_{\varphi} J_{\varphi}^{T}\left\{S_{v F}\right\} \\
J_{\varphi}^{\#}(q) \hat{S}_{q} & =S_{F \delta}+\gamma_{F 1} \int_{t_{0}}^{t} S_{F \delta}+\gamma_{F 2} \int_{t_{0}}^{t} \operatorname{sign}\left(S_{F \delta}\right)
\end{aligned}
$$

where $J_{\varphi}^{\#}(q)=\left(-\beta J_{\varphi} J_{\varphi}^{T}(q)\right)^{-1} J_{\varphi}$. Derivating (46) and multiply it by $S_{F \delta}^{T}$ becomes

$$
\begin{aligned}
S_{F \delta}^{T} \dot{S}_{F \delta} & =-\gamma_{F 2}\left|S_{F \delta}\right|-\gamma_{F 1} S_{F \delta}^{T} S_{F \delta}+S_{F \delta}^{T} \frac{d}{d t}\left(J_{\varphi}^{\#}(q) \hat{S}_{q}\right) \\
& \leq-\mu_{F}\left|S_{F \delta}\right|-\gamma_{F 1}\left\|S_{F \delta}\right\|^{2}
\end{aligned}
$$

where $\mu_{F}=\gamma_{F 2}-\varepsilon_{5}$, and $\varepsilon_{5}=\frac{d}{d t}\left[\left(J_{\varphi} J_{\varphi}^{T}(q)\right)^{-1} J_{\varphi} \hat{S}_{q}\right]$. If $\gamma_{F 2}>\varepsilon_{5}$, then a sliding mode at $S_{F \delta}(t)=0$ is induced at $t_{f} \leq \frac{\left|S_{F \delta}\left(t_{0}\right)\right|}{\mu_{F}}$, but $S_{F \delta}\left(t_{0}\right)=0$, thus $S_{F \delta}\left(t_{0}\right)=0$ is guaranteed $\forall t$.

Part III: Exponential convergence of tracking errors.

Part III.a: Visual tracking errors. Since a sliding mode exists for all time at $S_{s \delta}(t)=0$, then, we have

$$
S_{s}=S_{s d} \forall t \rightarrow \Delta \dot{x}_{s}=-\alpha \Delta x_{s}+S_{s}\left(t_{0}\right) e^{-\kappa_{s} t}
$$

this implies that the visual tracking errors locally tends to zero exponentially fast, implying that the robot end-effector converges to the desired image $x_{s d}$, with given velocity $\dot{x}_{s d}$.

Part III.b: Force tracking errors. Since a sliding mode at $S_{F \delta}(t)=0$ is induced for all time, this means $\Delta F=$ $\Delta F\left(t_{0}\right) e^{-\kappa_{F} t}$. Moreover, in [10] it is shown that the convergence of force tracking errors arises, thus $\lambda \rightarrow \lambda_{d}$ exponentially fast. QED.

Proof of Theorem2 : With the very same Lyapunov function of Theorem 1, we obtain the following time derivative, along trajectories of the closed loop of (32)-(33),

$$
\dot{V} \leq-K_{d}\left\|\hat{S}_{q}\right\|_{2}^{2}-\eta \beta\left\|S_{v F}\right\|+\left\|\hat{S}_{q}\right\| \psi-\dot{V}_{f}
$$

where

$$
\begin{aligned}
\dot{V}_{f} & =\sigma_{0} \hat{S}_{q}^{T}\left[z+\sigma_{01} \tanh \left(\xi_{f} \hat{S}_{q}\right)\right]-\sigma_{01} \hat{S}_{q}[-z h(\dot{q}) \\
& \left.+\alpha_{0}{ }^{-1} \sigma_{01}|\dot{q}| \tanh \left(\xi_{f} \hat{S}_{q}\right)\right] .
\end{aligned}
$$

In [11], [12] it was proved that $\dot{V}_{f}>0$, and $\left|\dot{V}_{f}\right|<\varepsilon_{4}$, $\varepsilon_{4}>0$. Then, $\dot{V}_{f}$ is positive de nite outside the hyperball $\rho_{0}=\rho_{0}\left(\hat{S}_{q}\right)=\left\{\hat{S}_{q} \mid V_{f} \geq 0\right\}$ with $\left\|\rho_{0}\right\| \leq \rho$, for $\rho>0$. Thus, if we choose $\xi_{f}$ large enough, preventing that the mechatronic system does not introduce high frequency from the term $\tanh \left(\xi_{f} \hat{S}_{q}\right)$, then (49) becomes

$$
\dot{V} \leq-K_{d}\left\|\hat{S}_{q}\right\|_{2}^{2}-\eta \beta\left\|S_{v F}\right\|+\left\|\hat{S}_{q}\right\| \psi+\rho_{0} .
$$

Afterwards, we proceed exactly as in proof of theorem 1, and it is therefore omitted. QED. 